\title{
High midplane accessibility stellarator windings for high-current toroidal accelerators
}

\author{
S. J. Marsh, a) J. Golden, and C. A. Kapetanakos \\ Plasma Physies Division, Naval Research Laboratory, Washington, DC 20375-5000 \\ David Anderson \\ Omicron Technology, Tnc. Madison, Wisconsin 53705
}

(Received 14 March 1988; accepted for publication 8 August 1988)

\begin{abstract}
We have developed two strong focusing field configurations that provide high midplane accessibility and therefore are compatible with presently contempiated extraction schemes for high-current, toroidal accelerators. When these windings are attached to a modified betatron accelerator, the bandwidth of the device is slightly smaller than the bandwidth of a modified betatron with unsplit stellarator windings. However, the bandwidth of the two new configurations is more than sufficient even for modest $(20-30 \mathrm{kA})$ winding currents.
\end{abstract}

\section{INTRODUCTIOR}

Stability analysis of the electron ring orbits over the last few years has shown that the confining properties of the modified betatron accelerator ${ }^{\mathrm{i}-8}$ can be substantially improved by adding strong focusing ${ }^{9-11}$ windings that carry modest current.

The windings that generate the strong focusing field, with poloidal multiplicity $l=2$, can be either of the stellarator ${ }^{9}$ type, i.e., four twisted wires with the current in adjacent wires fowing in opposite directions, or of the torsatron ${ }^{10,13}$. type, in which two twisted wires carry current in the same direction.

While improving the confining properties of the device, the strong focusing field makes the injection and trapping as well as the extraction of the bean from the magnetic field configuration substantially more involved.

Recently, two injection schemes ${ }^{10,12}$ have been developed for toroidal accelerators with strong focusing. In this paper we propose two modified stellarator winding configurtions that provide very high midplane accessibility and therefore are compatible with the presently contemplated radial extraction schemes.

Figure 1 (a) shows a three-period stellarator winding in the toroidal angle $\theta$, poloidal angle $\phi$ plane. By cutting these windings near $\phi=0$ and $\phi=2 \pi$, and connecting them as shown in Fig. $1(b)$, we obtain a configuration with the current fowing continuously through all the windings as well as the various jumpers that connect them. The parasitic fields generated by the jumpers are substantially reduced by two windbacks that run along the jumpers and carry half the current of the main windings. In an actual device the proposed split winding continuous stellarator configuration will look as shown in Fig. 2.

In the second, the modular, high midplane accessibility stellarator configuration the windings are cut again near $\phi=0$ and $\phi=2 \pi$ but are connected as shown in Fig. $3(\mathrm{~b})$. It should be noticed that the direction of the current in the jumpers that are located near $\phi=0$ is opposite to the direction of the current in the jumpers that are located near $\phi=2 \pi$. This provides partial cancellation of the parasitic

\footnotetext{
a) Sachs/Freeman Associates, Landover, MO 20785.
}

fields generated by the jumpers. Further reduction of the parasitic fields is obtained by two windbacks that run along the jumpers and carry current in the opposite direction. The magnitude of the current in each windback is one half of the current in the windings. In an actual device the proposed modular split winding stellarator configuration will look as shown in Fig. 4.

Although the two configurations appear to be substantially different, careful examination of Figs. 2 and 4 reveal that when the windbacks are included, both the magnitude as well as the direction of the current are identical in the two systems. This conclusion is also supported by the numerical studies of the ring dynamics in these two configurations.

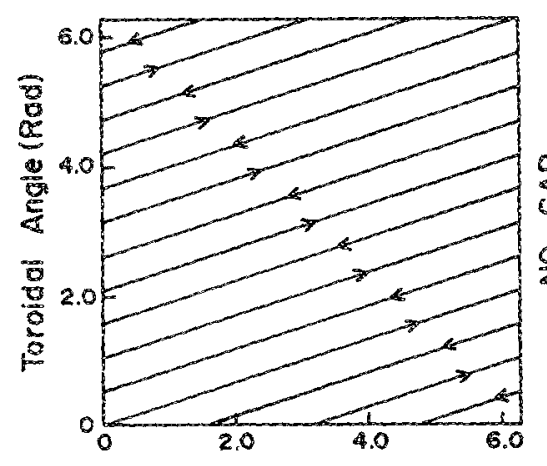

(a)

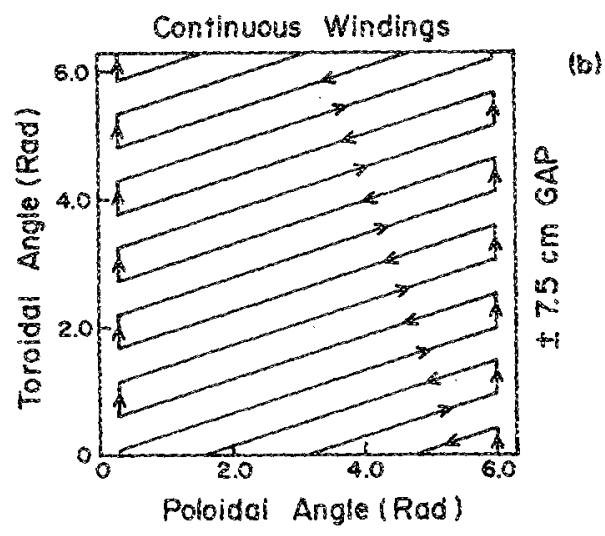

FIG. 1. Toroidal angle $\theta$, poloidal angle $\phi$ a plane for three-period steilaraior winding. (a) shows the unsplir stellarator windings and (b) shows the spilit windings in the continuous current configurations. The windbacks are not shown in this figure. 


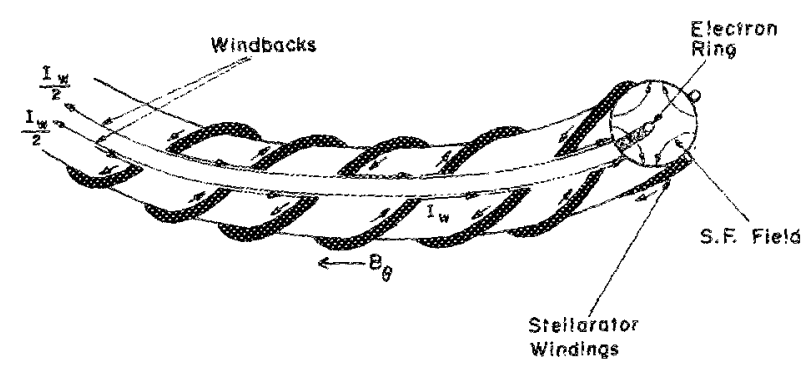

FIG. 2. Schematic of the split windings in the continuous current configuration. A though this figure shows left handed windings all the calculations have been performed for right-handed systems.

These studies have shown the ring orbits are identical when the various parameters of the two systems are selected to be the same.

\section{NUMERICAL RESULTS}

To determine the beneficial effect of these two split winding stellarator configurations on the confining properties of the modined betatron, we have carried out extensive numerical studies of the ring dynamics for a wide range of initial conditions. These studies have mainly focused on the transverse macroscopic electron ring orbits. A typical trans. verse electron ring orbit is shown in Fig. 5. A measure of the bandwidth of the device is the parameter $\delta$ that is defined as the distance from the injection position $(106 \mathrm{~cm}$ for all the data presented in this report) to the point where the beam

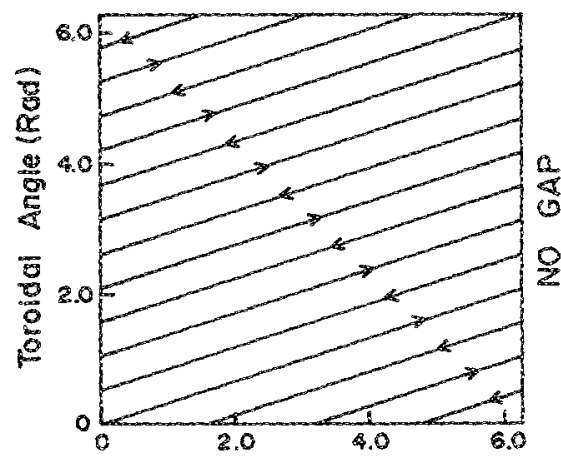

(a)

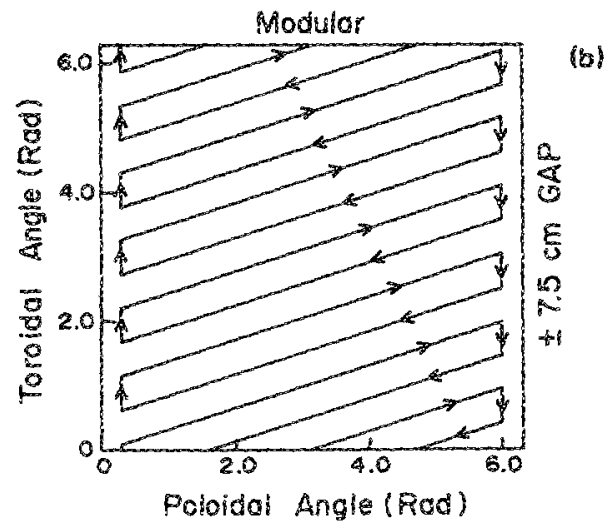

FIG. 3. Torodal angle $\theta$, poloidal angle $\phi$ plane for a three-period stellarator winding. (a) shows the unsplit stellarator windings and (b) shows the split windings in the modular contiguration. The windbacks russ along the jumpers but are not shown in this igure.

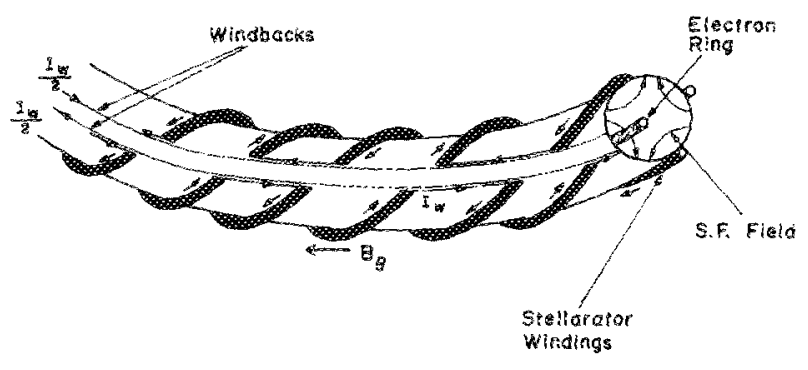

FIG. 4. Schematic of the split windings in the modular configuration.

orbit crosses the midplane $(z=0)$. Since $\delta$ is measured from the injection position, its value is negative when the ring orbit crosses the midplane between the inner wall of the torus and the injection position. For an injection position at $106 \mathrm{~cm}$ in a $100-\mathrm{crn}$ major radius chamber, we define the "useful bandwiath" as the range of the vertical magnetic field $B_{z}$ for which $\delta$ is between 0 and $\delta_{\max }$, where $\delta_{\max } \equiv$ inner wall tores radius + minor beam radius - injection position. Those orbits that cross the $z=0$ plane between the injection position and the outer wall of the torus are not of interest because the rings that move along these orbits cannot be moved near the minor axis without suffering severe losses on the injector.

To benchmark our results, we initially studied the varjation of $\delta$ with $B_{z}$ in a modified betatron with unsplit (regular) stellarator windings for four different windings currents. The macroscopic ring orbits have been obtained from the numerical integration of equations of motion. The magnetic fields of the stellarator-type windings have been calculated from the Biot-Savart law using flaments of infinitesimal radius. The continuous helical flaments and jumpers are approximated by a series of discrete segments, the number of which can be adjusted. The results for a four-windingperiod stellarator (eight-field period) are shown in Fig. 6 . The values of the various parameters for this figure are listed in Table I. It is apparent from these results that as the current through the windings increases, the curve rotates counterclockwise. For the lowest two winding currents 10 and 10 $\mathrm{kA}$ ) the slope of the curve is positive. This is exactly as expected for a space-charge dominated electron ring.

It has been shown previously that near the minor axis of the torus the linearized constant of the motion in a modifed betatron with torsatron windings is given by

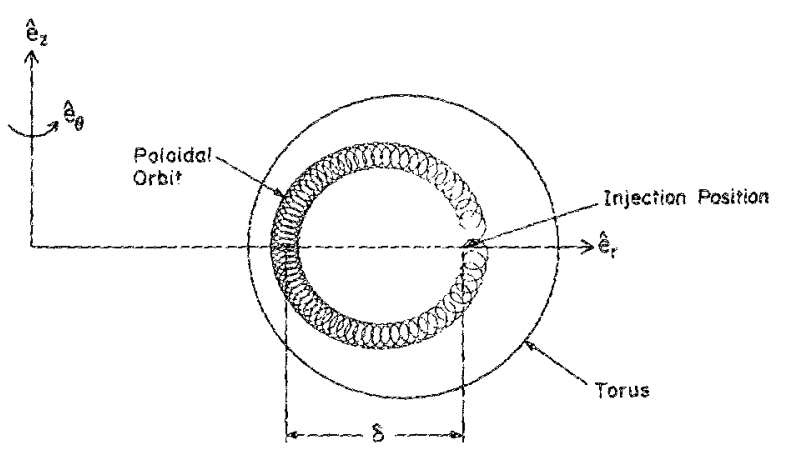

FIC. 5. Systen of coordinates and macroscopic (centroid) ring orbit in the transverse plane. 


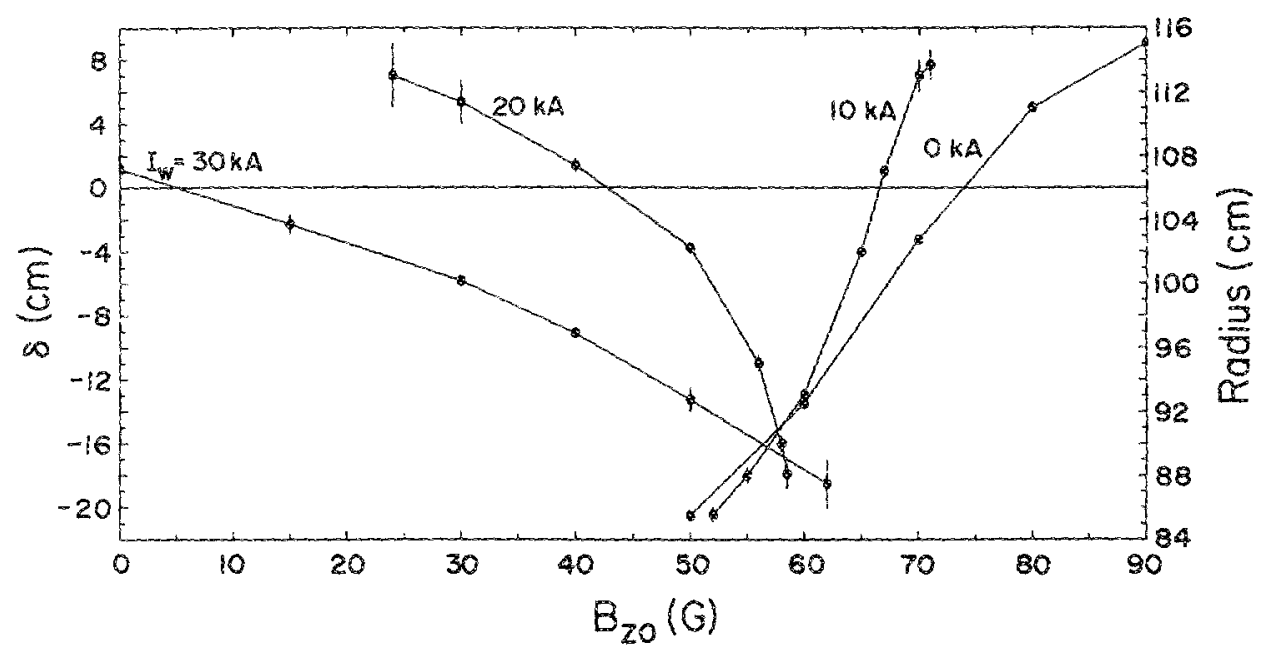

FIG. 6. Radial dimension $\delta$ of the macroscopic ring orbit in the transverse plane as a function of the vertical magnetic field $b_{20}$ for a modified betatron with conventional (unsplit) stellarator windings.

$$
k_{0}=q_{1}\left(\frac{X}{r_{0}}\right)^{2}+q_{2}\left(\frac{Z}{r_{0}}\right)^{2}-\frac{2 \delta p_{\theta}}{m r_{0}^{2} \Omega_{20}}\left(\frac{X}{r_{0}}\right)
$$

where

$$
\begin{aligned}
& q_{1}=1-n+n_{t}-n^{*}+2\left\langle P_{\theta}\right\rangle / m r_{0}^{2} \Omega_{20}, \\
& q_{2}=n+n_{t}-n^{*}, \\
& K_{0}=-\frac{2 \delta P_{\theta}}{m r_{0}^{2} \Omega_{00}}, \quad n_{t}=\frac{\left(\Omega_{\theta 0}^{\mathrm{ex}} \epsilon_{t}\right)^{2} r_{0} \alpha}{2 \Omega_{z 0}\left(-\hat{\Omega}_{\theta 0}+\beta_{\theta 0} \gamma_{0} \omega_{w 0}\right)},
\end{aligned}
$$

is the torsation field index, $n^{*}=2 v r_{0} c /\left(\gamma_{0}^{2} a^{2} \beta_{\theta 0} \Omega_{20}\right)$ and $\widehat{\Omega}_{\theta 0}<0$ is the combined toroidal field at $r_{0}$. The rest of the parameters are defined in Ref. 10.

For stellarator windings the coefficient $\Omega_{s 0}^{e x} \epsilon_{s}=2 \Omega_{s 0}^{e x} \epsilon_{t}$ and therefore for the same current in the windings the stellarator field index becomes

$$
n_{s}=\frac{4\left(\Omega_{s 0}^{\mathrm{ex}} \epsilon_{i}\right)^{2} \gamma_{0} \alpha}{2 \Omega_{20}\left(\Omega_{s 0}^{\mathrm{ex}}+\beta_{\theta 0} \gamma_{0} \omega \alpha_{\omega 0}\right)} .
$$

In Eq. (2) we have replaced $-\widehat{\Omega}_{\theta 0}=\Omega_{s 0}^{\text {ex }}$ since the zeroorder toroidal magnetic field generated by the stellarator windings is zero. Bquation (1) can be written as

$$
\left(\sqrt{q_{1}} \frac{X}{r_{0}}+\frac{K_{0}}{2 \sqrt{q_{1}}}\right)^{2}+q_{2}\left(\frac{Z}{r_{0}}\right)^{2}=K_{0}\left(1+\frac{K_{0}}{4 q_{1}}\right)
$$

and therefore the displacement of the center of the orbit $\Delta X$ fron the minor axis is

TABLE I. Various parameters common to all runs in Figs. 6-10.

$\begin{aligned} \text { Total beam energy (diode) } \gamma_{d} & =3.35 \\ \text { Beam kinetic energy } y & =1.75 \\ \text { Bean radius } r_{b} & =1.0 \mathrm{~cm} \\ \text { Beam current } & =4.92 \mathrm{kA} \\ \text { Torus major radius } r_{0} & =100 \mathrm{~cm} \\ \text { Torus minor radius } a & =16 \mathrm{~cm} \\ \text { Stellarator winding radius } & =20 \mathrm{~cm} \\ \text { Stellarator winding period } & =157 \mathrm{~cm} \\ \text { Centroid position at injection } & =106 \mathrm{~cm} \\ \text { Toroidal meagnetic feld at } r_{0}, z=0, B_{b_{b}} & =-500 \mathrm{G} \\ \text { External feld index } n & =0.5\end{aligned}$

$$
\frac{\Delta X}{r_{0}}=-\frac{K_{0}}{2 q_{1}}=\frac{2 \delta P_{\theta} / m r_{0}^{2} \Omega_{20}}{1-n+n_{s}-n^{*}+2\left\langle p_{\theta}\right\rangle / m r_{0}^{2} \Omega_{\infty 0}}
$$

Since the coefficient $\Omega_{s}^{e x} \epsilon_{s}$ is proportional to the current flowing in the stellarator windings, as the winding current increases, $n_{s}$ increases until $q_{1}\left(q_{1}\right.$ is less than $q_{2}$ for $n=0.5$ ) becomes zero. At this value of the winding current the curve of Fig. 6 becomes vertical. For even higher values of the current the reference electron at the ring centroid behaves like a single particle. This is manifested by the negative slope of the curves for 20 and $30 \mathrm{kA}$.

The curve for $30 \mathrm{kA}$ in Fig. 6 has been truncated at $B_{z}$ $=0$ because we are interested only in the useful bandwidth of the device. Actually, the 30-kA curve intersects the outer wall of the torus at $B_{x 0}=-55 \mathrm{G}$.

Figure 7 shows $\delta$ vs $B_{20}$ for three values of the current when the opening in the windings is $4 \mathrm{~cm}$ wide. This opening is centered around $z=0$. A new feature appears in this figure that was not present in Fig. 6. Both the 20-and 30-kA curves stop considerably short of the outer wall and the $20 \mathrm{kA}$ curve does not reach the inner wall. Within the range of the parameters considered, we have not found stable orbits in these regions. The ring centroid orbits change from stable to unstable with very small change in the value of the vertical magnetic field.

Figures 8 and 9 show $\delta$ vs $B_{x 0}$ with a 4-crn-wide gap and without a gap for 10 - and $20-\mathrm{kA}$ winding current. It appears that in the space-charge dominated regime (Fig. 8) the gap slightly increases the bandwidth of the device. However, in the single-particle regime (Fig. 9) the gap always reduces the bandwidth.

Figure 10 shows $\delta$ vs $B_{20}$ for 0 , 4 -, and 8 -cm-wide gaps when the current in the windings is $30 \mathrm{kA}$. It is apparent from these results that the bandwidth of the device is reduced as the width of the gap increases. For a 4-cm gap, the ring equilibrium position is located on the minor axis when $B_{20}=40 \mathrm{G}$. Therefore, the fractional bandwidth of the device is $\Delta B_{z} / B_{x 0}=(67-5) / 40=1.55$ or $155 \%$, which is more than sufficient.

Table II summarizes the useful bandwidth of the various configurations that have been presented in this report. 

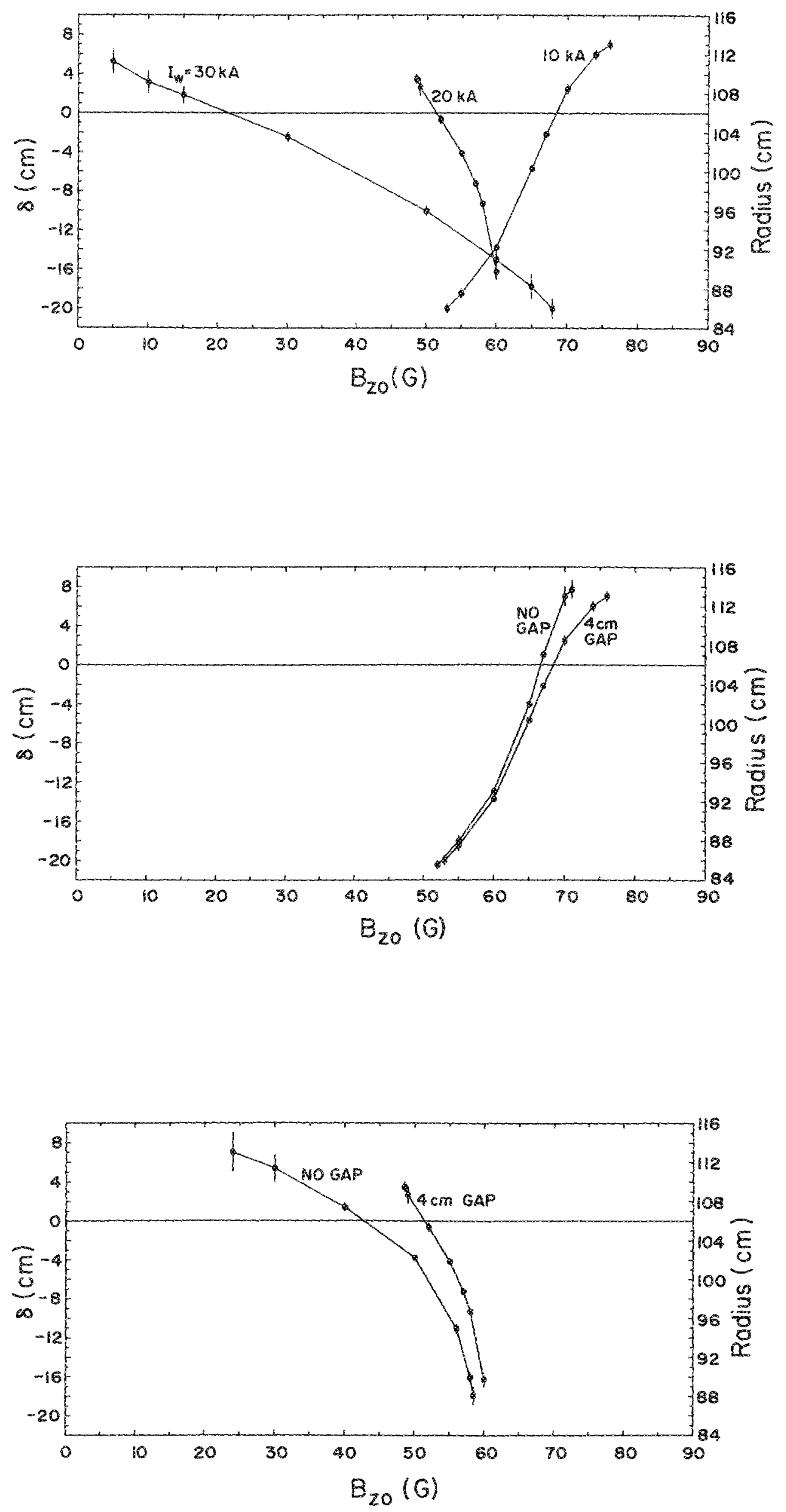

FIG. 7. Radial dimension $\delta$ of the macroscopic ring orbit in the transverse plane as a function of the vertical magnetic field $B_{x 0}$ for a modified betatron with a $4-\mathrm{cm}$ gap strong focusing modular windings. The results of the continuous windings are very similar.

FIG. 8. Variation of $\delta$ with $B_{30}$ for 0 and 4-cm gap windings. These resulis have been obtained with IO-kA winding cur. rent.

FIG. 9. As in Fig. 8 but with 20-kA winding current. 


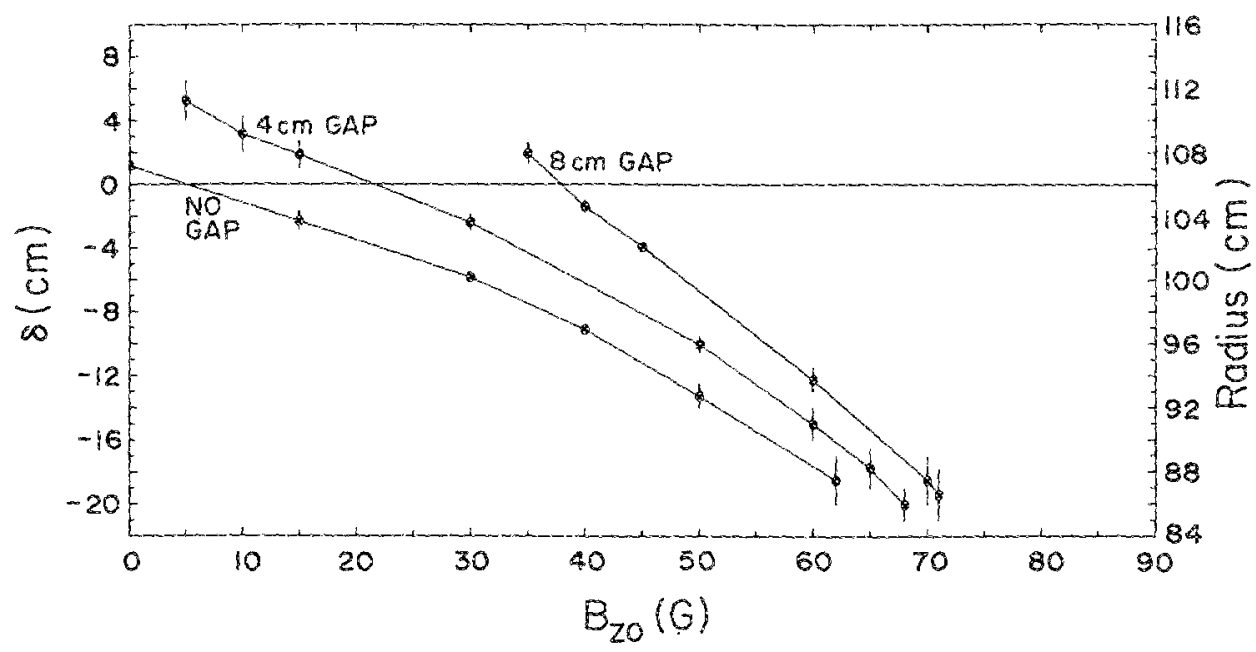

FIG. 10. Comparison of $\delta$ for three systems with $0-, 4-$, and $8-\mathrm{cm}$ gap windings. The current in the main windings is $30 \mathrm{kA}$.

The negative values of $\Delta B_{z}$ correspond to space-chargedominated equilibrium, i.e., when both $q_{1}$ and $q_{2}$ are negative. However, during acceleration $n^{*}$ is reduced and thus the ring has to cross the instability gap and probably will be lost.

\section{COACLUSIONS}

We have developed two strong focusing field configurations that provide high midplane accessibility and therefore are compatible with presently contemplated extraction schemes from high-current, toroidal accelerators. Aithough

TABLE II. Summary of useful bandwidths for Figs. 6-10.

\begin{tabular}{ccccc}
\hline $\begin{array}{c}\text { Winding } \\
\text { current } \\
(\mathrm{kA})\end{array}$ & $\begin{array}{c}B_{0} \\
(\mathrm{~cm})\end{array}$ & $\begin{array}{c}B_{20} \\
\text { injection }\end{array}$ & $\begin{array}{c}\text { inner wall } \\
(\mathrm{G})\end{array}$ & $\begin{array}{c}\text { Bandwidth } \\
4 B_{z}\end{array}$ \\
\hline 0 & 0 & 50 & 74 & $(\mathrm{G})$ \\
10 & 0 & 52 & 66 & -24 \\
10 & 4 & 53 & 68 & -14 \\
20 & 0 & 58 & 43 & -16 \\
20 & 4 & 60 & 48 & +15 \\
30 & 0 & 62 & 5 & +12 \\
30 & 4 & 68 & 22 & +57 \\
30 & 8 & 71 & 38 & +46 \\
\hline
\end{tabular}

the useful bandwidth of these configurations is smaller than the bandwidth of the unsplit stellatron, it is more than suffcient even for modest $(20-30 \mathrm{kA})$ winding current.

\section{ACKNOWLEOGMENTS}

This work was supported by Spawar 11-4, PMW 145P and ONR.

'P. Sprangle and C. A. Xapetanakos, J. Appl. Phys. 49, 1 (1978).

${ }^{2}$ C. A. Kapetanakos, P. Sprangle, D. P. Chernin, S. I. Marsh, and I. Haber, Phys. Fluids 26, 1634 (1983).

${ }^{3}$ D. Chernin and P. Sprangle, Part. Accel. 12, 85 (1982).

${ }^{4}$ W. Manheimer and J. Finn, Part. Accel. 14, 29 (1983).

${ }^{5}$ C. A. Kapetanakos and S. I. Marsh, Phys. Fluids 28, 2263 (1985).

'J. M. Grossman, T. M. Finn, and W. Manheimer, Phys. Fluids 29, 695 (1985).

${ }^{7}$ G. Barok and N. Rostoker, Phys. Fluids 26, 856 (1983)

${ }^{8}$ H. S. Uhm, R. C. Davidson, and J. Petilo, Phys. Fluids (1985).

${ }^{9}$ C. W. Roberson, A. Mondelli, and D. Chernin, Phys. Rev. Lett. 50, 507 (1983).

${ }^{11}$ C. A. Kaperanakos, D. Dialetis, and S. I. Marsh, Part. Accel. 1987; NRL Memo Report No. 5619 (1985).

"C. A. Kapetanakos, D. Dialetis, and S. J. Marsh, Nuel. Inst. Methods Phys. Res. 24, 805 (1987).

12y. Sprangle and C. A. Kapentanakos, Part. Accel. 18,203 (1986). 